\title{
Minimally Invasive Glaucoma Surgery with a New Ab-externo Subconjunctival Bypass - Current Status and Review of Literature
}

\author{
Henny JM Beckers ${ }^{1}$ and Leonard Pinchuk ${ }^{2}$ \\ 1. University Eye Clinic, Maastricht University Medical Center, Maastricht, The Netherlands; 2. InnFocus, Inc., \\ A Santen Company, Miami, Florida, USA
}

$\mathrm{T}$ he PRESERFLOTMMicroShunt (Santen, Osaka, Japan) is a new ab-externo, subconjunctival, minimally invasive glaucoma drainage device for surgical treatment of patients with early to advanced primary open angle glaucoma. It is made of a new polymer, 'SIBS' (poly[styrene-block-isobutylene-block-styrene]), and consists of an 8.5-mm long tube with an outer diameter of $350 \mu \mathrm{m}$ and a lumen diameter of $70 \mu \mathrm{m}$. Placement of the device is combined with intraoperative application of mitomycin C (MMC). The Microshunt received the CE Mark in 2012 but was only released for sale in the European market in 2019. Outside Europe, the device is currently being tested in Canada, Singapore, the USA and the Dominican Republic. Published evidence is still limited; however, study results to date show that a mean intraocular pressure (IOP) reduction of 30-55\% from baseline can be achieved, with a substantial reduction in glaucoma medications. Current studies also suggest that an MMC concentration of $0.4 \mathrm{mg} / \mathrm{mL}$ is more effective than $0.2 \mathrm{mg} / \mathrm{mL}$. Success rates (i.e., no failures [IOP $\geq 21 \mathrm{mmHg}$ or $<20 \%$ IOP reduction] with or without glaucoma medication) were found to vary between $80-100 \%$ at $1-2$ years of followup. Adverse events (e.g. hyphema, hypotony, shallow anterior chamber or choroidal effusion in 10-25\% of cases) were mild. They usually occurred within the first postoperative week and spontaneously resolved within 1-3 months. No long-term sight-threatening complications have been reported so far. Patients are usually satisfied with the procedure. Although more robust data are needed, these first studies suggest that the PRESERFLO Microshunt is a promising new, minimally invasive procedure for glaucoma.

\section{Keywords}

PRESERFLOTM Microshunt, InnFocus Microshunt, minimimally invasive glaucoma surgery

Disclosure: Henny Beckers has received research grants from Alcon Benelux and Santen, and financial support from Alcon, Santen, Glaukos and Allergan. Len Pinchuk is Founder and CSO of InnFocus, Inc., A Santen Company.

Review Process: Double-blind peer review.

Compliance with Ethics: This study involves a review of the literature and did not involve any studies with human or animal subjects performed by any of the authors.

Access: This article is freely accessible at tOuChOPHTHALMOLOGY.COm.

(C) Touch Medical Media 2019

Authorship: The named authors meet the International Committee of Medical Journal Editors (ICMJE) criteria

for authorship of this manuscript, take responsibility for the integrity of the work as a whole, and have given final approval for the version to be published.

Received: 31 May 2019

Accepted: 22 July 2019

Citation: European Ophthalmic Review. 2019;13(1):27-30

Corresponding Author: Henny JM Beckers,

University Eye Clinic, Maastricht University Medical

Center, P. Debyelaan 25, 6229 HX Maastricht, The

Netherlands. E: henny.beckers@mumc.nl

Support: No funding was received for

the publication of this article.
The PRESERFLOTMMicroShunt (Santen, Osaka, Japan), formerly known as the InnFocus Microshunt (Santen, Osaka, Japan), is a minimally invasive glaucoma drainage device, intended to shunt aqueous humour from the anterior chamber (AC) of the eye to a filtration bleb, formed under the conjunctiva and Tenon's capsule. The device was initially designed for use in patients with early to advanced primary open angle glaucoma and is placed via an ab-externo approach with intraoperative application of mitomycin C (MMC). The Microshunt received the CE Mark in 2012 and is currently only commercially available in Europe. Randomised controlled studies on safety and efficacy are underway in Europe, Canada, Singapore, the USA and the Dominican Republic.

\section{MicroShunt material and design}

The MicroShunt is made of a novel polymer, 'SIBS' (poly[styrene-block-isobutylene-block-styrene]; Figure 1). ${ }^{1,2}$ It is a unique stretchy material, designed specifically for long-term implantation in the body; it is expected to outlive the patient. Another important feature of the material is that it provokes minimal inflammation and encapsulation when compared with commonly used materials such as silicone rubber and polypropylene. Lastly, the material is thermoformable, giving it the advantage of assuming the shape of the globe without a tendency to return to its original shape, as do thermoset materials such as silicone rubber. Silicone rubber tubing slowly straightens over time, therefore requiring that it be covered with a patch graft to prevent erosion through the conjunctiva. SIBS has no tendency to straighten, which eliminates the need for a patch graft.

SIBS was first used on Boston Scientific's (Natick, Massachusetts, USA) TAXUS ${ }^{\circledR}$ coronary artery stent, where a SIBS matrix, impregnated with the antiproliferative drug paclitaxel, is coated on the stent struts to deliver and release the drug in the coronary arteries as a means of preventing arterial restenosis. TAXUS, with its SIBS coating, has been used in the body since 1999. ${ }^{3}$

The MicroShunt design consists of an 8.5-mm long SIBS tube with an outer diameter of $350 \mu \mathrm{m}$ and a lumen diameter of $70 \mu \mathrm{m}$. It is a valveless tube that does not require temporary tube occlusion. The lumen diameter is sufficiently large to allow passage of sloughed endothelial cells (approximately 40-50 $\mu \mathrm{m}$ in diameter), yet sufficiently small to prevent clinically significant postoperative hypotony. The lumen diameter was approximated from the Hagan-Poiseuille equation and confirmed by a series of rabbit eye implants. ${ }^{4.5}$ With these design characteristics, the MicroShunt creates a transscleral gradient of $5-10 \mathrm{mmHg}$ at the physiological flow rate of aqueous 
Figure 1: SIBS structure formula

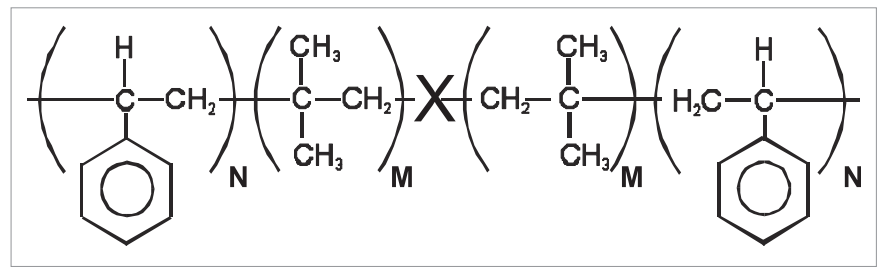

SIBS = poly(styrene-block-isobutylene-block-styrene).

humour ( 2.5 $\mu \mathrm{l} / \mathrm{min})$, which should lead to a controlled postoperative intraocular pressure (IOP) reduction with minimal hypotony.

The device is designed with a $1.1 \mathrm{~mm}$ wingspan 'fin' which is located halfway down the tube, which, during implantation, fixes the device in a 1-mm wide scleral pocket, thereby preventing the device from migrating into the eye. The fin also prevents leakage around the tube and helps orient the device bevel-up in the $\mathrm{AC}$, preventing the lumen from being blocked by debris or iris tissue. When correctly placed, the distal end of the Microshunt is located 6-mm posterior to the limbus. This posterior placement directs aqueous humour more posteriorly than trabeculectomy, which may possibly eliminate the thinner-walled anterior blebs that can occur with the latter procedure.

\section{MicroShunt implantation procedure}

The implantation procedure is shown in Figure 2. Subconjunctival or subTenon's anaesthesia is given at the surgeon's discretion. An approximately 5 -mm wide fornix-based conjunctival/Tenon's flap is created, after which a deep sub-Tenon's pocket (6-8 mm) is formed. A temporal-nasal approach is preferred to decrease the risk of damaging the superior rectus muscle. Wet field cautery is applied to prevent bleeding. Several corneal Lasik shields (usually three), soaked in $0.2-0.4 \mathrm{mg} / \mathrm{mL}$ of $\mathrm{MMC}$, are placed within the conjunctival/Tenon's flap for 2-3 minutes, after which, the shields are removed, and the ocular tissue carefully rinsed with 10-20 mL of balanced saline solution. After marking the sclera $3 \mathrm{~mm}$ from the limbus, a 1-mm deep scleral pocket is made using a triangular knife. A 25 gauge needle is introduced into the pocket and a scleral needle track is created into the AC. The Microshunt is inserted through the pocket and track, bevel-up, into the AC. When correctly placed, the MicroShunt should bisect the angle without touching the endothelium or the iris (Figure 3). The fin is tucked in the scleral pocket. Flow is established by gently applying pressure on the eye or flushing balanced saline solution through a side-port. Another method is to flush balanced saline solution through a 23 gauge cannula placed over the distal end of the tube. After checking for flow, Tenon's and conjunctiva are carefully pulled up (taking care not to tuck the distal end of the tube into Tenon's capsule) and sutured in a watertight fashion (suture material ad libitum).

After surgery, a light pressure patch is applied for the first postoperative day, after which, treatment commences with topical steroids (and if desired, topical antibiotic treatment), usually 4-6 times daily, slowly tapered over the course of several weeks to months. Figure 4 shows the bleb of two successfully operated patients after 3 years.

\section{Current evidence}

The MicroShunt received the CE Mark in 2012 but was only launched in Europe in 2019. Published evidence is therefore, still limited and includes pioneering studies that helped define the Microshunt, the preferred implantation procedure, and the method and concentration of MMC administration. ${ }^{6,7}$
Table 1 shows 5-year results of a first cohort of 23 trabeculectomystage eyes with primary open angle glaucoma. ${ }^{7.8}$ Nine of these patients underwent a combination Microshunt procedure with cataract surgery. MMC of $0.4 \mathrm{mg} / \mathrm{mL}$ concentration was used in all cases. The results show IOP outcomes in the low teens, that are maintained during followup with a substantial reduction in glaucoma medications for 5 years. Two-year results from a European/Dominican Republic multicentre study, presented the 2019 World Glaucoma Congress (manuscript in preparation), are shown in Table $2 .{ }^{9}$ This study revealed better IOP and medication outcomes with 67 patients treated with $0.4 \mathrm{mg} / \mathrm{mL} \mathrm{MMC}$ as compared to 58 patients treated with $0.2 \mathrm{mg} / \mathrm{mL}$ MMC. However, the better outcomes in the $0.4 \mathrm{mg} / \mathrm{mL} \mathrm{MMC}$ group may demonstrate an increased risk of complications (e.g. shallow/flat AC, transient hypotony, bleb leakage). In both groups, visual acuity (VA) remained stable after 2 years of follow-up, with patients usually regaining their preoperative VA within the first few weeks.

\section{Adverse events}

Adverse events (summarised from all studies and abstracts on efficacy and safety of the Microshunt at the time of this writing) as a result of the surgical procedure, occurred in 10-25\% of cases. .2, $^{2-11}$ They included hyphema (<10\%), numerical hypotony (10-16\%), shallow AC (4-13\%), choroidal detachment or effusion ( $<9 \%$ ), and typically occurred within the first postoperative week then spontaneously resolving within 1-3 months. Surgical failures (5-10\%) tended to occur within the first year and were usually due to bleb fibrosis requiring re-operation. Approximately two-thirds of the bleb revisions were treated by removing scar tissue from the bleb with re-establishment of flow through the Microshunt. The remaining surgical failures required trabeculectomy, or placement of a new Microshunt or a large drainage tube. Bleb needling was performed in $2-10 \%$ of cases, usually within 9 months of follow-up. Pressure failures (defined as IOP $\geq 21 \mathrm{mmHg}$ or $<20 \%$ reduction in IOP on two consecutive follow-up visits after 3 months) occurred in $2-10 \%$ of eyes within the first postoperative year and afterwards at a rate of $1-5 \%$ per year. Success rates (no failures, with or without glaucoma medication) varied between $80-100 \%$ at $1-2$ years of follow-up. There were no reported long-term sight-threatening complications (chronic hypotony, vitreous haemorrhage, light perception loss, hypotony maculopathy, etc).

\section{Discussion}

The PRESERFLO Microshunt is made of a unique biomaterial that is stretchy but also sufficiently rigid to be implanted in a straightforward, ab-externo procedure. The surgical technique does not require dissection of a scleral flap (as does trabeculectomy), no sclerectomy or iridectomy, and there is no special skill required to properly place flap sutures with the desired tension. It is also possible to reposition the Microshunt if placed incorrectly where a second or even a third scleral needle track can be created without causing hypotony. Postoperative interventions are minimal (e.g. no suture lysis); however, postoperative monitoring is required, especially in cases with an increased risk of postoperative inflammation where these eyes may require additional steroids and at times needling or bleb revision to prevent failure. Postoperative visual recovery is usually fast, in most cases returning to preoperative levels within the first few weeks (manuscript in preparation). The impact on quality of life of patients seems therefore mild.

The aforementioned studies show that a mean IOP reduction of 30$55 \%$ from baseline can be achieved, with a substantial reduction in the mean number of glaucoma medications per patient. In addition, these studies suggest that an MMC concentration of $0.4 \mathrm{mg} / \mathrm{mL}$ is more effective than $0.2 \mathrm{mg} / \mathrm{mL}$. An explanation for this finding might be 

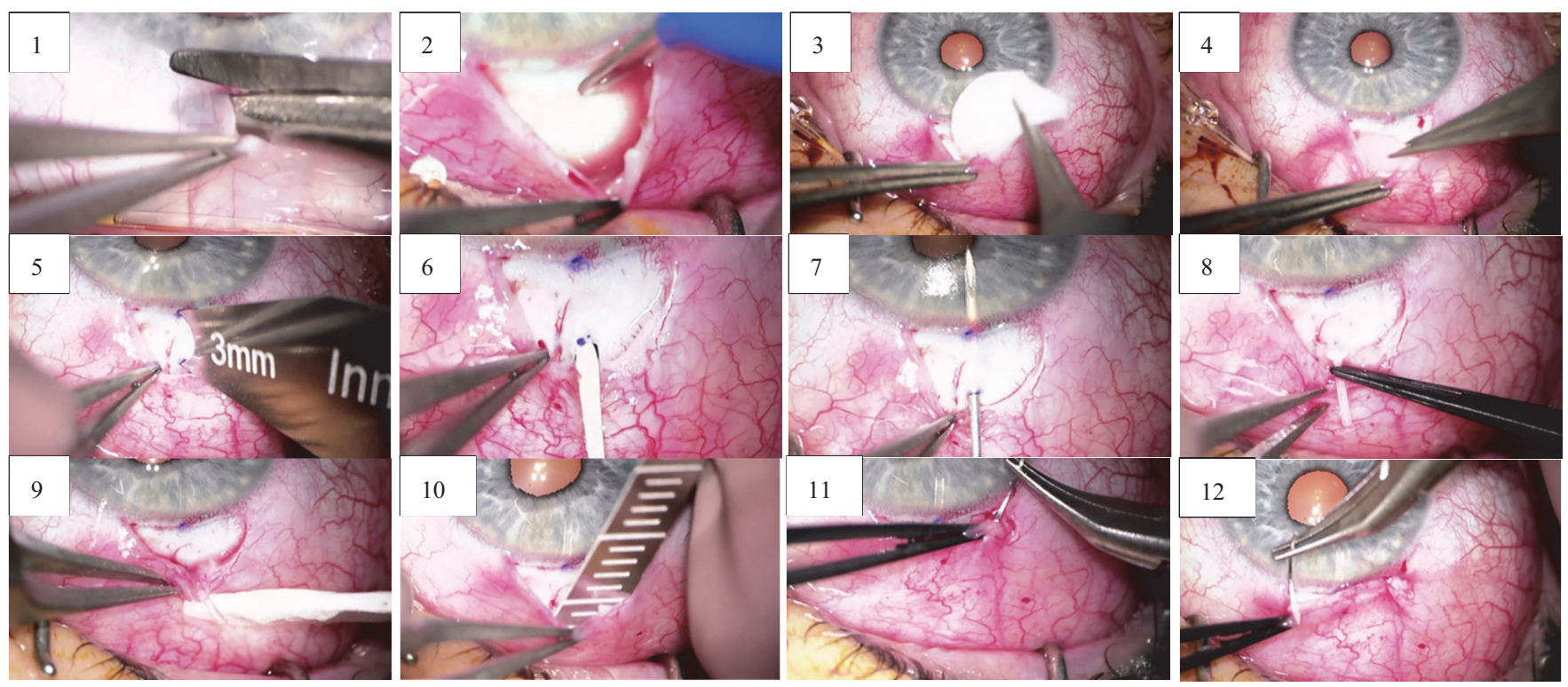

1. Creation of a fornix-based conjunctival/Tenon's flap: 2. Wet field cautery is applied to prevent bleeding: 3 . Lasik shields soaked in MMC are placed underneath the conjunctival/ Tenon's flap: 4. The shields are removed and the ocular tissues are rinsed with balanced saline solution. 5 . The sclera is marked, 3 mm from the limbus: 6 . A $1 \mathrm{~mm}$ scleral incision (pocket) is made: 7 . A 25 gauge scleral needle track is created: 8. The Microshunt is inserted through the needle track into the AC, the fin tucked in the scleral pocket: 9 . Flow is checked; 10. Tenon and conjunctiva are carefully pulled up with the tube kept flush to the sclera; 11. The wound is sutured in a watertight fashion.

Figure 3: 360-degree anterior segment optical coherence tomography view showing a correctly placed Microshunt

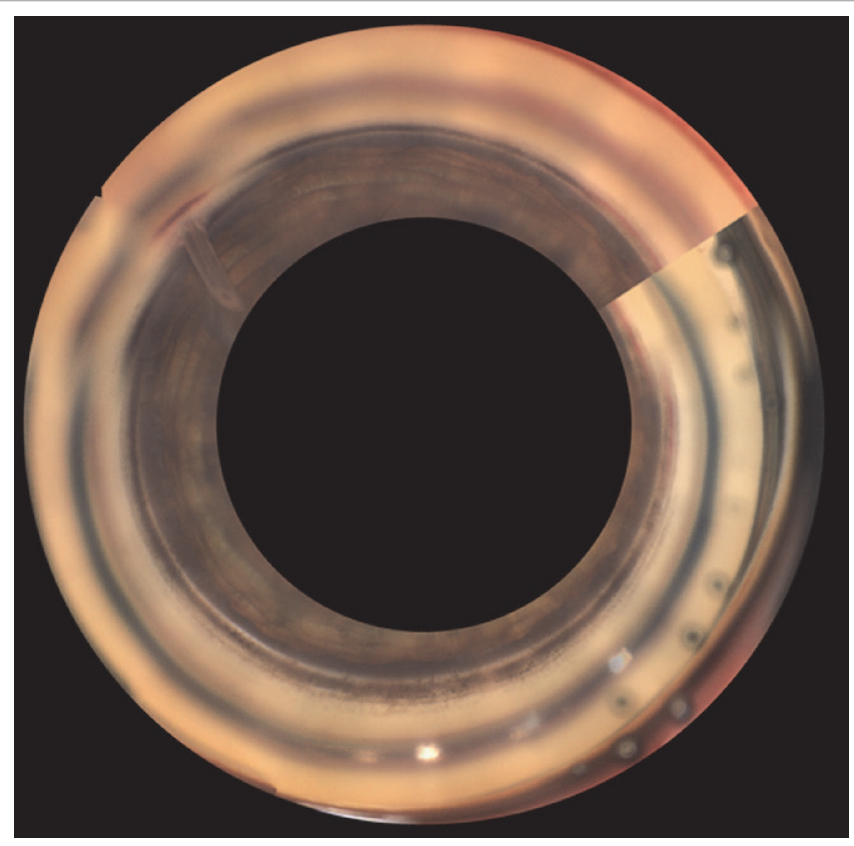

that the Microshunt distal end is approximately $6 \mathrm{~mm}$ posterior to the limbus, forming a more posterior bleb as compared to trabeculectomy. The conjunctiva and Tenon's are thicker in a more posterior bleb, which would prevent the bleb roofs from forming pin-holes and reduce the risk of endophthalmitis. However, these thicker blebs which are less elevated may also demonstrate a higher risk of failure.

The aforementioned studies did not show long-term sight-threatening adverse events, which indicate that Microshunt placement is a safe procedure for patients with open angle glaucoma. There is no published evidence yet with this device in angle closure patients.
Figure 4: Bleb aspect of two patients, both 3 years after successful Microshunt implantation
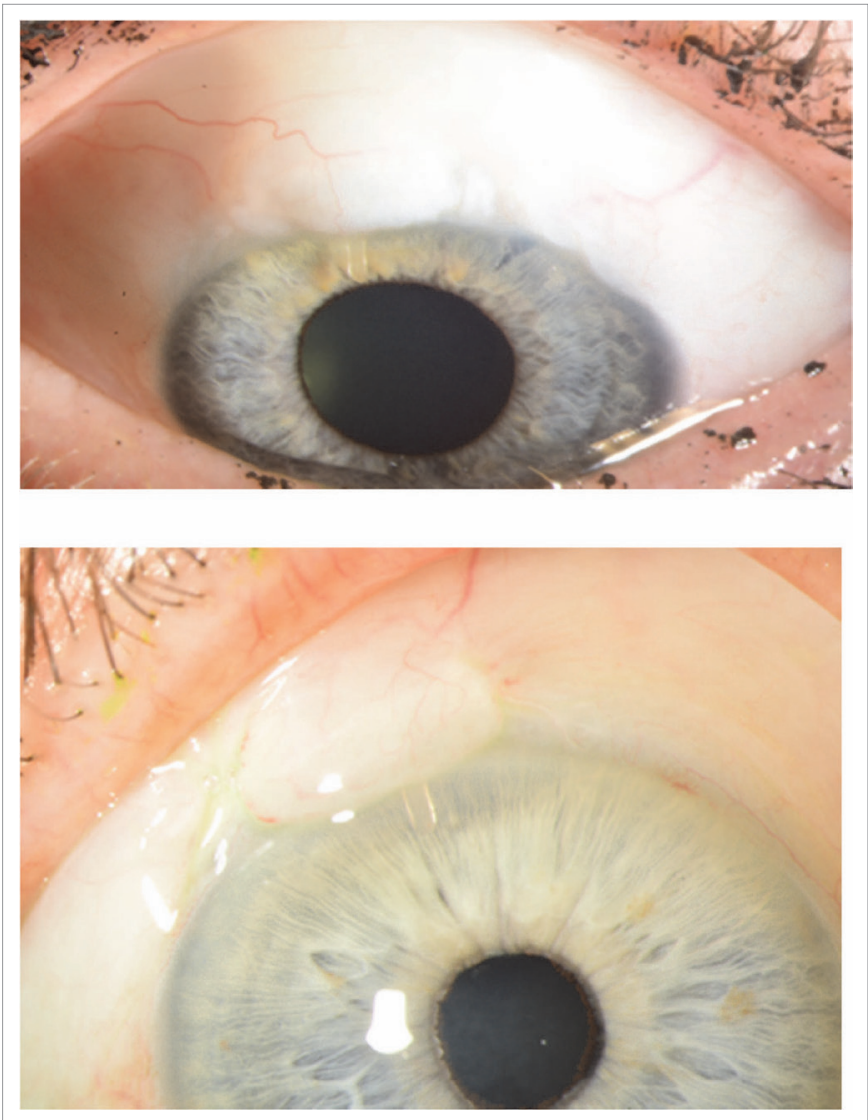

\section{Conclusion}

The PRESERFLO Microshunt provides a promising, new, minimally invasive procedure that may be indicated for patients with severe primary open angle glaucoma and may even serve as an alternative to primary 
Table 1: Five-year results of a cohort of 23 patients receiving Microshunt implantation with intraoperative application of $0.4 \mathrm{mg} / \mathrm{mL}$ mitomycin $\mathrm{C}^{7,8}$

\begin{tabular}{|l|l|l|l|l|}
\hline Time & n (9 combo) & $\begin{array}{l}\text { Qualified } \\
\text { success* }\end{array}$ & $\begin{array}{l}\text { IOP } \\
(\mathrm{mmHg})\end{array}$ & $\begin{array}{l}\text { Number of } \\
\text { medications per } \\
\text { patient }\end{array}$ \\
\hline Pre-op & 23 & $\mathrm{n} / \mathrm{a}$ & $23.8 \pm 5.3$ & $2.4 \pm 0.9$ \\
\hline Year 1 & 23 & $100 \%$ & $10.7 \pm 2.8$ & $0.3 \pm 0.8$ \\
\hline Year 2 & 22 & $91 \%$ & $11.9 \pm 3.7$ & $0.4 \pm 1.0$ \\
\hline Year 3 & 22 & $95 \%$ & $10.7 \pm 3.5$ & $0.7 \pm 1.1$ \\
\hline Year 4 & 14 & $87 \%$ & $12.3 \pm 3.7$ & $0.5 \pm 1.0$ \\
\hline Year 5 & 18 & $87 \%$ & $11.4 \pm 4.9$ & $0.5 \pm 1.1$ \\
\hline
\end{tabular}

$* I O P>6$ and $\leq 14 \mathrm{mmHg}$ with a reduction from baseline $\geq 20 \%$ ).

Nine of these patients also underwent phacoemulsification with implantation of an intraocular lens.

Combo = in combination with phacoemulsification; IOP = intraocular pressure.

trabeculectomy. Because of its safety, it may be used earlier in the treatment paradigm, as an alternative to long-term glaucoma medication, or in patients who are too early for end-stage filtering treatments, such as trabeculectomy or large drainage valves. However, as indicated in a multicentre study, ${ }^{9}$ the treatment may fail, and further studies are required to identify patients who will benefit from the procedure. In general, more robust data from clinical trials and cost-effectiveness
Table 2: Two-year results from the European/Dominican Republic multicentre study ${ }^{9}$

\begin{tabular}{|l|l|l|l|l|l|}
\hline & Time & $\mathrm{n}$ & IOP $(\mathrm{mmHg})$ & $\begin{array}{l}\text { Number of } \\
\text { medications } \\
\text { per patient }\end{array}$ & $\begin{array}{l}\text { Patients off } \\
\text { medication, } \\
\%\end{array}$ \\
\hline \multirow{2}{*}{$\begin{array}{l}\mathbf{0} 2 \mathrm{mg} / \mathrm{mL} \\
\mathrm{MMC}\end{array}$} & Pre-op & 58 & $22.7 \pm 4.4$ & $2.4 \pm 1.3$ & - \\
\cline { 2 - 6 } & Year 1 & 55 & $14.8 \pm 5.0$ & $0.7 \pm 1.1$ & 64.3 \\
\cline { 2 - 6 } & Year 2 & 50 & $14.8 \pm 4.5$ & $0.8 \pm 1.1$ & 59.6 \\
\hline \multirow{2}{*}{$\begin{array}{l}\text { M.4 mg/mL } \\
\text { MMC }\end{array}$} & Pre-op & 67 & $22.2 \pm 4.0$ & $2.1 \pm 1.2$ & - \\
\cline { 2 - 6 } & Year 1 & 59 & $12.9 \pm 3.7$ & $0.3 \pm 0.7$ & 84.8 \\
\cline { 2 - 6 } & Year 2 & 53 & $13.0 \pm 3.4$ & $0.2 \pm 0.6$ & 85.5 \\
\hline
\end{tabular}

The result show a less favourable outcome in terms of IOP reduction and reduction in glaucoma medications, for the patients who received the Microshunt that were treated with $0.2 \mathrm{mg} / \mathrm{mL} M M C$ when compared to the patients treated with $0.4 \mathrm{mg} / \mathrm{mL} \mathrm{MMC}$. $I O P=$ intraocular pressure; $M M C=$ mitomycin $C$.

studies are required. Currently, the Microshunt is being tested in Europe, Canada, Japan, Singapore and Dominican Republic. A multicentre study in the USA, comparing the Microshunt to primary trabeculectomy in patients who are refractory to medication, has recently completed enrolment. ${ }^{12}$ It is expected that the Microshunt will be commercialised in the USA in 2020. A cost-effectiveness study comparing the Microshunt to primary trabeculectomy is underway in the Netherlands. ${ }^{13} \square$
1. Pinchuk, L, Riss, I, Batlle JF, et al. The development of a micro-shunt made from poly (styrene-block-isobutyleneblock-styrene) to treat glaucoma. J Biomed Mater Res B App Biomater. 2017:105:211-21

2. Pinchuk L, Riss I, Batlle JF, et al. An Ab-Externo Minimally Invasive Aqueous Shunt Comprised of a Novel Biomateria. In: Ahmed I, Samples JR. New Concepts in Glaucoma Surgery Series; Kugler Publications, Amsterdam, The Netherlands (ISSN 2589-7632), vol. 1. In press, 2019

3. Pinchuk L, Wilson GJ, Barry JJ, et al. Medical applications of poly(styrene-block-isobutylene-block-styrene) ("SIBS"). Biomaterials. 2008:29:448-60

4. Arrieta EA, Aly M, Parrish II RK, et al. Clinicopathologic correlations of poly(styrene-b-isobutylene-b-styrene) glaucoma drainage devices of different internal diameters in rabbits. Ophthalmic Surg Lasers Imaging. 2011:42:338-45.
5. Fantes F, Acosta AC, Carraway J, et al. An independent GIP evaluation of a new glaucoma drain, the MIDI. Invest Ophthalmol Vis Sci. 2006:47:3547.

6. Riss I, Batlle J, Pinchuk L, et al. One-year results on the safety and efficacy of the InnFocus Microshunt ${ }^{T M}$ depending on placement and concentration of mitomycin C. J Fr Ophtalmol. 2015;38:855-60

7. Batlle JF, Fantes F, Riss I, et al. Three-year follow-up of a novel aqueous humor microshunt. J Glaucoma. 2016;25:e58-65.

8. Batlle JF, Alburquerque R, Corona Peralta A, et al. Four- and five-year results of the Microshunt in patients with primary openangle glaucoma. Presented at: APAO, Bangkok, Thailand, 2019.

2. García-Feijoó J, Batlle JF, Riss I, et al. A 2-year pooled analysis of the MicroShunt in patients with primary open-angle glaucoma (POAG): 0.2 versus $0.4 \mathrm{mg} / \mathrm{mL}$ mitomycin $\mathrm{C}$-anC) guaucoma Poster presented at World Glaucoma Congress (WGC).
Melbourne, Australia, 2019

10. Riss I, Aptel F, Beckers $\mathrm{H}$, et al. Interim 1-year outcomes following MicroShunt Glaucoma Drainage System implantation: multicentre Phase 4 study. Paper presented at World Ophthalmology Congress (WOC) Barcelona, Spain, 2018

11. Beckers HJM, Kujovic-Aleksov S, Webers CAB, et al. One-year results of a three-site study of the InnFocus Microshunt. Acta Ophthalmol. 2017;95:28-9.

12. Clinical Trials Gov. InnFocus Microshunt Versus Trabeculectomy Study (IMS). ClinicalTrials.gov Identifier: NCT01881425. Available at: https://clinicaltrials.gov/ct2/show/NCT01881425 (accessed 24 July 2019).

13. Clinical Trials Gov. Cost-effectiveness of InnFocus Microshunt Implantation vs. Trabeculectomy ClinicalTrials gov Identifier: NCT03931564. Available at: https://clinicaltrials gov/ct2/show/ NCT03931564 (accessed 24 July 2019). 